\title{
Effects of sharp vorticity gradients in two-dimensional hydrodynamic turbulence
}

\author{
Kuznetsov, E.A.; Naulin, Volker; Nielsen, Anders Henry; Juul Rasmussen, Jens
}

Published in:

Physics of Fluids

Link to article, DOI:

$10.1063 / 1.2793150$

Publication date:

2007

Document Version

Publisher's PDF, also known as Version of record

Link back to DTU Orbit

Citation (APA):

Kuznetsov, E. A., Naulin, V., Nielsen, A. H., \& Juul Rasmussen, J. (2007). Effects of sharp vorticity gradients in two-dimensional hydrodynamic turbulence. Physics of Fluids, 19(10), 105110. https://doi.org/10.1063/1.2793150

\section{General rights}

Copyright and moral rights for the publications made accessible in the public portal are retained by the authors and/or other copyright owners and it is a condition of accessing publications that users recognise and abide by the legal requirements associated with these rights.

- Users may download and print one copy of any publication from the public portal for the purpose of private study or research.

- You may not further distribute the material or use it for any profit-making activity or commercial gain

- You may freely distribute the URL identifying the publication in the public portal 


\title{
Effects of sharp vorticity gradients in two-dimensional hydrodynamic turbulence
}

\author{
E. A. Kuznetsov \\ P. N. Lebedev Physical Institute, 53 Leninsky Ave., 119991 Moscow, Russia and L. D. Landau Institute \\ for Theoretical Physics, 2 Kosygin str., 119334 Moscow, Russia \\ V. Naulin, A. H. Nielsen, and J. Juul Rasmussen \\ Optics and Plasma Research Department, OPL-128, Ris $\phi$ National Laboratory, Technical University \\ of Denmark, P.O. Box 49, DK-4000 Roskilde, Denmark
}

(Received 12 December 2006; accepted 24 August 2007; published online 24 October 2007)

The appearance of sharp vorticity gradients in two-dimensional hydrodynamic turbulence and their influence on the turbulent spectra are considered. We have developed the analog of the vortex line representation as a transformation to the curvilinear system of coordinates moving together with the divorticity lines. Compressibility of this mapping can be considered as the main reason for the formation of the sharp vorticity gradients at high Reynolds numbers. For two-dimensional turbulence in the case of strong anisotropy the sharp vorticity gradients can generate spectra which fall off as $k^{-3}$ at large $k$, resembling the Kraichnan spectrum for the enstrophy cascade. For turbulence with weak anisotropy the $k$ dependence of the spectrum due to the sharp gradients coincides with the Saffman spectrum, $E(k) \sim k^{-4}$. We have compared the analytical predictions with direct numerical solutions of the two-dimensional Euler equation for decaying turbulence. We observe that the divorticity is reaching very high values and is distributed locally in space along piecewise straight lines, thus indicating strong anisotropy, and accordingly we find a spectrum close to the $k^{-3}$ spectrum. (C) 2007 American Institute of Physics. [DOI: 10.1063/1.2793150]

\section{INTRODUCTION}

This paper is concerned with investigations of twodimensional (2D) hydrodynamical turbulent flows. In particular, we study the formation and dynamics of very sharp vorticity gradients and their influence on the energy spectrum in the enstrophy cascade regime. There are two kinds of well-known 2D turbulent spectra. The first one was suggested by Kraichnan ${ }^{1}$ in 1967 , it corresponds to the enstrophy cascade directed to the small-scale region where viscous dissipation becomes essential. The Kraichnan spectrum has, up to a logarithmic factor (see Ref. 2), a power law dependence for the intermediate wave numbers between source and sink (the inertial interval), $E(k) \sim \eta^{2 / 3} k^{-3}$, where $\eta$ is the enstrophy dissipation rate. [Recall that $2 \mathrm{D}$ turbulence additionally is characterized by an inverse energy cascade towards large-scales leading to the Kolmogorov dependence $E(k) \sim k^{-5 / 3}$ (see, e.g., Ref. 1)]. The second spectrum suggested by Saffman $^{3}$ in 1971 has a different power dependence, $E(k) \sim k^{-4}$. According to Saffman vorticity discontinuities will form in decaying 2D turbulence (in the absence of viscosity) because fluid elements with different values of vorticity will be driven close together by the flow. Due to vorticity conservation the appearance of discontinuities will respect the conservation of all other invariants involving the vorticity, $\int \omega^{n} d S, n=2,3,4, \ldots$. Accounting for a finite viscosity Saffman considers the "discontinuities" to have a small width $\delta$, which results from the balance between inertial and viscous forces. At high-Reynolds number this width is assumed to be very small as compared to the length along the discontinuities, $L$, which may be assumed to be of the same order as the characteristic energy-containing length scale. Under the assumption of isotropy and a dilute distribution of discontinuities Saffman suggested that the energy spectrum at large $k$ could be constructed as a superposition of the spectra from the individual discontinuities resulting in $E(k) \sim k^{-4}$

From the first sight, the spectra obtained by Kraichnan and Saffman appear like two different results, but indeed, as we show in this paper, it is possible to establish some connection between them. This may be seen from the Fourier transform of a step function. Let us assume that the vorticity has a jump $\Gamma=\Gamma(x)$ along the line $y=0$. At first we neglect effects connected with the finite jump width, $\delta$, and bending of the line. Then we may write

$$
\frac{\partial \omega}{\partial y}=\Gamma \delta(y) .
$$

Hence it is immediately seen that the Fourier transform will have a power-law falloff at large $k$, i.e., inversely proportional to $k_{y}$ multiplied by some function of $k_{x}$ due to the dependence of $\Gamma$ on $x$. If we neglect the dependence on $k_{x}$ replacing it by some constant, we immediately obtain an energy spectrum with a power dependence, which appears to be similar to the Kraichnan spectrum, $E(k) \sim k^{-3}$. This is an important conjecture demonstrating that a spectrum similar to the Kraichnan spectrum, which is often observed in high resolution numerical simulations, may be related to vorticity jumps, which could be considered as possible candidates for singularities in ideal fluids in two dimensions. However, for ideal 2D fluids, i.e., in the framework of the Euler equations, such singularities are not possible since the vorticity is a Lagrangian invariant. Its gradient might grow very large, but cannot become infinite in finite time. This is a consequence 
of the fundamental results obtained by Wolibner, ${ }^{4}$ Kato,${ }^{5}$ and Yudovich $^{6}$ (see also the reviews in Refs. 7 and 8). The supremum of the vorticity gradient is bounded from above by a double exponential law with a characteristic time of about the vortex turnover time. This dependence is very sharp from the physical point of view. Indeed, if one considers the double exponential function $\exp \left[\exp \left(\omega_{0} t\right)\right]$ as an estimate [compare with Eq. (10)] then within two turnover times, $\omega_{0} t=2$, the maximal possible amplification of the vorticity gradient is larger than $10^{3}$ ( $\omega_{0}$ is the vorticity). It should also be noted that for viscous fluids, i.e., in the framework of the Navier-Stokes equation, the initial smoothness of the solution will remain for all times as proven first by Olga Ladyzhenskaya ${ }^{9}$ many years ago. Moreover, this is valid also for the Navier-Stokes equations with hyperviscosity. ${ }^{10}$

It is necessary to mention some examples of $2 \mathrm{D}$ flows in ideal fluids presented by Yudovich, ${ }^{6}$ where the appearance of weaker singularities (vorticity is allowed to be discontinuous but bounded) are possible, however, they are formed in infinite time. Another approach based on the numerical analysis of the complex singularities for the inviscid flow with twomode initial conditions showed that the width of its analyticity strip follows a $\ln (1 / t)$ law at short times. ${ }^{11,12}$ Additionally many numerical experiments for 2D turbulence (see Refs. 13-16) show that the Saffman spectrum is formed with a good accuracy in the initial stage, before the excitation of the long-scale coherent vortices. The high-resolution numerical simulation performed by Legras et al. ${ }^{17}$ demonstrated the power dependence $k^{-3.5}$. Analytical calculations presented by Gilbert ${ }^{18}$ using spiral structures provide a power dependence with exponent between -3 and -4 (see also Refs. 19 and 20). In particular, we would like to point to the interesting work by Ohkitani, ${ }^{21}$ where by means of the Weiss decomposition ${ }^{22}$ it was shown that the so-called $h$ regions ( $h$ hyperbolic, i.e., regions where straining is dominating over vorticity) give the spectrum $k^{-3}$, i.e., coinciding up to a logarithmic factor with the Kraichnan spectrum; the contribution from the $e$ regions (e elliptic, i.e., vorticity dominated regions) yields a behavior like the Saffman spectrum $\sim k^{-4}$. Similar observations were reported by Chen et al. ${ }^{23}$ and by Do-Khac et al. ${ }^{24}$ who applied a wavelet analysis to separate 2D turbulent flow into regions having different dynamical behaviors. The appearance of a power law type spectrum in the short-wave region has been connected with different physical mechanisms like vortex merging ${ }^{25-27}$ and vortex stripping ${ }^{28,29}$ which give a certain confirmation of the original idea of Saffman. ${ }^{3}$

In the present paper we present qualitative physical arguments in favor of the formation of sharp vorticity gradients in the 2D Euler equations for smooth initial conditions. The main idea in the description of $2 \mathrm{D}$ flows is to use the Lagrangian invariance of the vorticity. Kuznetsov and Ruban ${ }^{30}$ (see also Ref. 31) developed a new description for threedimensional vortical flows-the so-called vortex line representation (VLR). This representation is based on the mixed Lagrangian-Eulerian description and connected with movable vortex lines. The VLR, which is a mapping to a curvilinear system of coordinates, turns out to be compressible. This is considered to be the main reason for steepening of gradients and ultimate breaking in hydrodynamics. Here we shall demonstrate how this approach can be modified for the 2D Euler hydrodynamics. The main observation is that for 2D flows the curl of the vorticity, sometimes referred to as the divorticity, ${ }^{14,21,22}$ represents a frozen-in field, i.e., it satisfies the same equation as, e.g., the equation for the magnetic field in ideal 2D magnetohydrodynamics (MHD). Therefore the generalization to the 2D Euler equations becomes straightforward. In the local case, as it was demonstrated for the 2D Euler equations in Ref. 32, the vorticity plays the role of a Lagrangian coordinate and the other variable coincides with the Cartesian coordinate, say, $x$. In terms of these variables the 2D Euler equations transform into equations of motion for a layered fluid, similar to a stratified fluid, where each layer is labeled by its vorticity $\omega$. In terms of the new variables the "new" hydrodynamics becomes compressible. The derivative $y_{\omega}$, as a function of time and coordinate $x$, plays the role of density of each layer, and it is proportional to the width between two neighboring layers with closed vorticity contours.

Another aim of this paper is revisiting the energy spectra for $2 \mathrm{D}$ turbulence with emphasis on the angle distribution, following the arguments of Saffman connected with vorticity discontinuities. Using the stationary phase method we demonstrate that the contribution from one discontinuity is very anisotropic; it has a sharp angular peak along the direction perpendicular to the discontinuity. In the peak the energy spectrum falls-off like $k^{-3}$ at large $k$. After averaging over angles in the case of isotropic turbulence the spectrum coincides with the Saffman spectrum. ${ }^{3}$

In order to investigate whether the spectrum at large $k$ is defined by sharp vorticity gradients, we have performed numerical experiments on decaying turbulence based on a direct numerical solution of the $2 \mathrm{D}$ Euler equations. In the turbulent state when the formation of power law tails in the spectrum is observed, we examined the structure of the divorticity $(\mathbf{B}=\nabla \times \omega \hat{z})$. We found that the divorticity is distributed very sharply in space concentrated on a random net of lines. In our opinion, these results can be interpreted in favor of the Saffman mechanism for the formation of $2 \mathrm{D}$ turbulent spectra due to discontinuities.

The remaining of the paper is organized as follows: In Sec. II we derive the equation for the divorticity and apply the VLR to investigate the development of steep vorticity gradients in 2D flows. Then in Sec. III we discuss the energy spectrum in 2D turbulence and its relation to steep vorticity gradients. The relation between the spectrum and the developing steep vorticity gradients is examined in Sec. IV by direct numerical solutions of the 2D Euler equations. Finally, Sec. V contains our concluding remarks.

\section{TWO-DIMENSIONAL ANALOG OF THE VORTEX LINE REPRESENTATION}

Consider a 2D ideal fluid, described by the Euler equation for the vorticity $\omega(x, y, t)$,

$$
\frac{d \omega}{d t} \equiv \frac{\partial \omega}{\partial t}+(\mathbf{v} \cdot \nabla) \omega=0, \quad \nabla \cdot \mathbf{v}=0,
$$


where the velocity field $\mathbf{v}$ defines the vorticity, $\omega=(\nabla$ $\times \mathbf{v}) \cdot \hat{z}=\partial v_{y} / \partial x-\partial v_{x} / \partial y$. Equation (1) shows that the vorticity is a Lagrangian invariant advected by the fluid, i.e., the vorticity is invariant along a fluid particle trajectory defined as the solution of the system of ordinary differential equations (ODEs),

$$
\frac{d \mathbf{r}}{d t}=\mathbf{v}(\mathbf{r}, t),\left.\quad \mathbf{r}\right|_{t=0}=\mathbf{a} .
$$

Let us introduce the divergence-free vector field $\mathbf{B}$ with the components

$$
B_{x}=\frac{\partial \omega}{\partial y}, \quad B_{y}=-\frac{\partial \omega}{\partial x},
$$

i.e., $\mathbf{B}=\nabla \times \omega \hat{z}$. It is easily seen that this vector is tangent to the line $\omega(x, y)=$ const because the vorticity gradient $\nabla \omega$ $=\left(\partial_{x} \omega, \partial_{y} \omega\right)$ is normal to this line. The equation of motion for B can be obtained from Eq. (1) after differentiating with respect to coordinates,

$$
\frac{\partial \mathbf{B}}{\partial t}=\nabla \times[\mathbf{v} \times \mathbf{B}] .
$$

Thus, the vector $\mathbf{B}$ constitutes a frozen-in quantity, and it is often called the divorticity (see Ref. 14). In terms of the substantial derivative, Eq. (3) can be rewritten as

$$
\frac{d \mathbf{B}}{d t}=(\mathbf{B} \cdot \nabla) \mathbf{v} \equiv \frac{1}{2}[\omega \hat{z} \times \mathbf{B}]+\hat{S} \mathbf{B} .
$$

Here the first term on the rhs describes the rotation of the divorticity vector with the angular velocity $-\omega / 2$ and the second term is responsible for stretching of the divorticity lines, where

$$
\hat{S}_{i k}=\frac{1}{2}\left(\frac{\partial v_{k}}{\partial x_{i}}+\frac{\partial v_{i}}{\partial x_{k}}\right)
$$

is the stress tensor. Hence the divorticity length $|\mathbf{B}|$ will locally increase due to stretching when

$$
\frac{1}{2} \frac{d \mathbf{B}^{2}}{d t}=(\mathbf{B} \cdot \hat{S} \mathbf{B})>0 .
$$

It is interesting to note that this equation resembles the equation for the vorticity square $\omega^{2}$ for three-dimensional turbulence, where the stretching of vorticity lines enhances the vorticity, cf. Ref. 33. Increasing (or decreasing) the divorticity field, based on Eq. (6), is not sufficient to clarify the physical mechanism for its growth. As is seen from Eq. (3) only one velocity component, $\mathbf{v}_{n}$, normal to the vector $\mathbf{B}$ changes the field $\mathbf{B}$. In this case the tangential component $\mathbf{v}_{\tau}$ (parallel to $\mathbf{B}$ ) plays a passive role providing the incompressibility condition, $\nabla \cdot \mathbf{v}_{\tau}+\nabla \cdot \mathbf{v}_{n}=0$. This observation is the key point for introducing the VLR for the three-dimensional Euler equations (see, e.g., Ref. 31). To construct the analog of VLR for the 2D Euler equations we consider new Lagrangian trajectories, given by $\mathbf{v}_{n}$,

$$
\frac{d \mathbf{r}}{d t}=\mathbf{v}_{n}(\mathbf{r}, t),\left.\quad \mathbf{r}\right|_{t=0}=\mathbf{a} .
$$

The solution of these ODEs defines a new mapping

$$
\mathbf{r}=\mathbf{r}(\mathbf{a}, t),
$$

which is different from that given by Eq. (2). In terms of this mapping the divorticity equation (3) can easily be integrated,

$$
\mathbf{B}(\mathbf{r}, t)=\frac{\left(\mathbf{B}_{\mathbf{0}}(\mathbf{a}) \cdot \nabla_{a}\right) \mathbf{r}(\mathbf{a}, t)}{J},
$$

where $\mathbf{B}_{\mathbf{0}}(\mathbf{a})$ is the initial divorticity, $J$ is the Jacobian of the mapping (8), $J=\partial(x, y) / \partial\left(a_{x}, a_{y}\right)$. According to the definition of this mapping the Jacobian is not fixed, it may change in time and space. In other words, the mapping $\mathbf{r}=\mathbf{r}(\mathbf{a}, t)$, as a change of variables, represents a compressible mapping. This means that the divorticity lines can be compressed. In this approach the velocity of motion of divorticity lines is simply the normal velocity $\mathbf{v}_{n}$.

It should be noted that this approach in slightly different form was suggested in Ref. 32. There the basis is the mixed Lagrangian-Eulerian description when all desired quantities are considered as functions of vorticity $\omega$ (or any other Lagrangian invariant) and a Cartesian coordinate $x$.

The representations (7)-(9) do not work at singular points where the $\mathbf{B}$-field vanishes, $\mathbf{B}(\mathbf{r}(t), t)=0$, and where, respectively, the normal velocity is not defined. For the vorticity $\omega$ these points correspond to the maximal, minimal or saddle points. It is observed that the null points are advected by the fluid, but the velocity $\mathbf{v}$ at these points is defined through the $\mathbf{B}$-field by inverting the Laplacian operator, $\mathbf{v}$ $=-\Delta^{-1} \mathbf{B}$. The null-points for the normal vector field, $\mathbf{n}(\mathbf{r})$, represent topological singularities. Topological constraints as additional conditions to the systems (7)-(9) are written as integrals of the vector field $\mathbf{n}(\mathbf{r})$ along a loop enclosing the null-points (see, e.g., Ref. 34),

$$
\oint(\nabla \varphi \cdot d \mathbf{r})=2 \pi m,
$$

where $\varphi$ is the angle between the vector $\mathbf{n}$ and the $x$-axis and $m$, the topological charge, is an integer equal to the total number of turns of the vector $\mathbf{n}$, while passing around the closed contour with the null-point inside it (see also Ref. 32). For instance, at $X$-points or $O$-points $m= \pm 1$.

As well-known from gas dynamics, compressibility of the mapping is the main cause for steepening and ultimately breaking, resulting in the formation of sharp gradients for the velocity and density of the gases. This happens in finite time and in the general situation the singularity first appears at one separate point, i.e., it may be related to collapse. In gas dynamics this process is completely characterized by the mapping determined by the transition from the Eulerian to the Lagrangian description. Vanishing of the Jacobian corresponds to the emergence of a singularity. For threedimensional incompressible Euler equations compressibility of the VLR is a possible reason for the appearance of infinite vorticity in one separate point resulting in breaking of vortex lines. The first study of vortex-line breaking for three- 
dimensional integrable hydrodynamics with the Hamiltonian $\int|\boldsymbol{\omega}| d \mathbf{r}$ was performed by Kuznetsov and Ruban. ${ }^{35}$ Recent numerical experiments ${ }^{36,37}$ have confirmed the possibility of this type of scenario.

The Jacobian in the denominator of the expressions (9) can tend to zero implying the increasing of the divorticity. We do not see any restrictions why this process should be forbidden. In 2D hydrodynamics compressibility of the mapping guarantees only compression of divorticity lines corresponding to the formation of sharp gradients for the vorticity. However, the breaking process for 2D flows of ideal fluids can only take place in infinite time. As it was shown by Wolibner, ${ }^{4}$ Kato, ${ }^{5}$ and Yudovich ${ }^{6}$ collapse as a process of singularity formation in finite time is forbidden for 2D ideal fluid flows. In accordance with these results the distance $\rho(t)$ between two fluid particles occurs to be bounded from below by the double exponential dependence (see also the reviews in Refs. 7 and 8),

$$
\rho(t) \geq L \exp \left[-\log \left(L / \rho_{0}\right) \exp \left(C|\omega|_{\left.L^{\infty} t\right)},\right.\right.
$$

where $\rho_{0}$ is the initial separation, $L$ is the characteristic size of the fluid domain, $|\omega|_{L^{\infty}}$ is the supremum of the vorticity, and $C$ is a constant of order 1 . This estimate shows the impossibility of vanishing separation, $\rho(t)$, in finite time. For the vorticity gradient — or the divorticity — this means boundedness from above, also by a double exponential dependence, ${ }^{7,8}$

$$
|\nabla \omega(t)|_{L^{\infty}} \leq\left|\nabla \omega_{0}\right|_{L^{\infty}} \exp \left[\frac{C\left|\omega_{0}\right|_{\alpha}}{C_{1}\left|\omega_{0}\right|_{L^{\infty}}}\left\{\exp \left(C_{1} t\left|\omega_{0}\right|_{L^{\infty}}\right)-1\right\}\right] .
$$

Here $\left|\omega_{0}\right|_{\alpha}$ is the Hölder norm of the initial vorticity and $C_{1}$ is another constant of order 1. The rhs of Eq. (10) defines the time $T=\left|\omega_{0}\right|_{L^{\infty}}^{-1}$ coinciding with the characteristic turnover time. At $t=0$ the estimate (10) becomes precise. A significant deviation of $|\nabla \omega(t)|_{L^{\infty}}$ from the initial value $\left|\nabla \omega_{0}\right|_{L^{\infty}}$ should be expected for $t>T$. At these times, in principle, we cannot exclude some intermediate asymptotics with a sharp growth different from the estimate (10). In Sec. IV we present numerical results for the evolution of $|\nabla \omega(t)|_{L^{\infty}}$ and its relation to Eq. (10).

The most important point in our opinion is that the tendency of vorticity steepening indeed exist. For ideal fluids the appearance of infinite vorticity gradients can happen only in infinite time. It is, however, possible to imagine that this process may be accelerated in the presence of external forces driving the turbulence.

\section{2D SPECTRUM}

In the previous section we presented arguments in favor of the formation of sharp gradients of the vorticity in 2D Euler flows. In the following we will suppose that this process is possible and consider how it can effect the form of the turbulent spectrum. For 2D turbulence, in the presence of finite viscosity and external forcing, we will assume that the sharp vorticity gradients have a finite value, which is defined from the balance between inertial and viscous terms. The value of the gradient is related to a characteristic scale length $\delta=(|\nabla \omega| /|\omega|)^{-1}$, which determines the width of the corresponding localized stripes of strongly enhanced amplitude in the $|\nabla \omega|$ field. At high Reynolds number the width $\delta$ will be much less than the characteristic (energy-containing) scale $L$. In the turbulent state such enhanced stripes in $|\nabla \omega|$ are naturally forming a set of vorticity jumps with random positions of their centers, random forms and random distributions over angles. Our aim is to calculate the contribution to the spectrum from such sharp jumps. We will be interested in the region of wave numbers $k$ lying between $L^{-1}$ and the inverse width $\delta^{-1}$,

$$
L^{-1} \ll k \ll \delta^{-1} .
$$

To simplify the problem all jumps are supposed to be concentrated on pieces of straight lines (finite intervals) with the vorticity jump vanishing at the endpoints of the intervals. As we will see later this simplification is not so essential. The result, which we obtain, will also account for bends of the stripes.

To find the spectrum we need to calculate the Fourier transform of the pair correlation function,

$$
F(\mathbf{r})=\langle\omega(\mathbf{x}) \omega(\mathbf{x}+\mathbf{r})\rangle,
$$

where angle brackets means average over the ensemble of jumps. Hence the energy density spectrum $\boldsymbol{\epsilon}(\mathbf{k})$ is given by the standard expression,

$$
\epsilon(\mathbf{k})=\frac{F_{k}}{2 k^{2}}=\frac{\overline{\left|\omega_{k}\right|^{2}}}{8 \pi^{2} S k^{2}},
$$

where $\omega_{k}$ is the Fourier transform of the vorticity $\omega(\mathbf{r})$,

$$
\omega_{k}=\int \omega(\mathbf{r}) e^{-i(\mathbf{k r})} d \mathbf{r}, \quad \omega(\mathbf{r})=\frac{1}{(2 \pi)^{2}} \int \omega_{k} e^{i(\mathbf{k r})} d \mathbf{k},
$$

where the overbar denotes average with respect to random variables and $S$ is the average area, which is assumed to be sufficiently large.

Consider first one jump with the center at $\mathbf{r}_{\mathbf{0}}=\left(x_{0}, y_{0}\right)$ oriented along the $y$-axis. Approximating the jump by a step discontinuity we have for the $y$-derivative of $\omega$,

$$
\frac{\partial \omega}{\partial y}=\Gamma(x) \delta\left(y-y_{0}\right)+\text { regular terms } .
$$

Here $\Gamma(x)$ is a continuous function of $x$ inside the interval $\left[x_{1}, x_{2}\right]$ vanishing at the end points $x=x_{1,2}$ and equal to zero outside the interval.

Hence, the Fourier transform from the singular part of $\omega$ is given by the integral

$$
\omega_{k}=-\frac{i}{k_{y}} e^{-i k_{y} y_{0}} \int_{x_{1}}^{x_{2}} \Gamma(x) e^{-i k_{x} x} d x,
$$

where $\mathbf{k}=\left(k_{x}, k_{y}\right)$. This is the contribution from one jump. If we assume that the jumps are not very densely distributed, they may be considered "independent" and the spectrum for the whole ensemble of jumps is obtained by a superposition of the spectra from the individual jumps, i.e., from the summation with respect to all jumps which results in 


$$
\omega_{k}=-\sum_{\alpha} \frac{i}{\left(\mathbf{k} \cdot \mathbf{n}_{\alpha}\right)} e^{-i\left(\mathbf{k} \cdot \mathbf{n}_{\alpha}\right) y_{0 \alpha}} \int_{x_{1 \alpha}}^{x_{2 \alpha}} \Gamma_{\alpha}(x) e^{-i\left(\mathbf{k} \cdot \tau_{\alpha}\right) x} d x .
$$

Here we have introduced two unit vectors: Normal $\mathbf{n}_{\alpha}$ and tangent $\boldsymbol{\tau}_{\alpha}\left(\mathbf{n}_{\alpha}^{2}=\boldsymbol{\tau}_{\alpha}^{2}=1,\left(\mathbf{n}_{\alpha} \cdot \boldsymbol{\tau}_{\alpha}\right)=0\right)$ characterizing the orientation of the $\alpha$ th jump. The coordinates $x_{1 \alpha}, x_{2 \alpha}, y_{0 \alpha}$ together with the two unit vectors define completely the positions of the jumps.

To find the enstrophy spectrum one needs to perform the average of $\left|\omega_{k}\right|^{2}$ with respect to all random variables. Assuming the coordinates of the jumps to be randomly distributed uniformly in space, the first average gives

$$
\overline{\left|\omega_{k}\right|^{2}}=N\left\langle\frac{1}{(\mathbf{k} \cdot \mathbf{n})^{2}}\left|\int_{x_{1}}^{x_{2}} \Gamma(x) e^{-i(\mathbf{k} \cdot \tau) x} d x\right|^{2}\right\rangle .
$$

$N$ is the number of jumps in area $S$, angle brackets means the average with respect to both $x_{1}, x_{2}$, and angle distribution.

We are interested in short-wave asymptotics of the spectrum, $k L \gg 1$, then the integrand in Eq. (13) represents a rapidly varying function of $x$, and the integral can be estimated by means of the method of stationary phase. This method can be applied for all angles except for a narrow cone of angles, $\theta_{k}\left(\theta_{k}\right.$ is the angle between the vectors $\mathbf{n}$ and k) where $k L \theta_{k} \leq 1$. In this region the integral can be considered as constant which results in the following form for the energy distribution (before angle averaging!):

$$
\epsilon_{1}(\mathbf{k}) \approx \frac{n}{8 \pi^{2} k^{4}}\left\langle(\bar{\Gamma} l)^{2}\right\rangle, \quad \theta_{k} \leq \theta_{0} \equiv(k L)^{-1},
$$

where $n=N / S$ is the density of jumps and

$$
\bar{\Gamma} l=\int_{x_{1}}^{x_{2}} \Gamma(x) d x, \quad l=x_{2}-x_{1}, \quad\langle l\rangle=L .
$$

For angles $\theta_{k}$ lying far from $\theta_{0} \equiv(k L)^{-1}$ the integral in Eq. (13) is estimated by means of the method of stationary phase. However, the leading order, proportional to $(\mathbf{k} \cdot \boldsymbol{\tau})^{-1}$, gives zero input because $\Gamma\left(x_{1,2}\right)=0$. Therefore one needs to keep the next order approximation that gives

$$
\epsilon_{2}(\mathbf{k}) \approx \frac{N}{4 \pi^{2} k^{2}} \frac{\left\langle\left(\Gamma^{\prime}\right)^{2}\right\rangle}{(\mathbf{k} \cdot \mathbf{n})^{2}(\mathbf{k} \cdot \boldsymbol{\tau})^{4}}, \quad \theta_{k} \gg(k L)^{-1},
$$

where $\Gamma^{\prime}$ is the derivative of $\Gamma$ taken at the endpoints $x_{i}$. This formula demonstrates singular behavior for $\epsilon(\mathbf{k})$ for angles $\theta_{k}$ close to 0 and $\pi / 2$ (as well as to $\pi$ and $-\pi / 2$ ). At small angles $\theta_{k} \leq(k L)^{-1}$ this expression has to be matched with Eq. (14). For the angle range close to $\pi / 2$ the integral in Eq. (15) should be cut-off due to the bending of the line of the jump. This factor switches on at angles $\left|\theta_{k}-\pi / 2\right| \sim(k a)^{-1}$, where $a$ is a characteristic bending length of the jump (roughly of the order of $L$ ). Thus, the energy density distribution $\epsilon(\mathbf{k})$ has a very narrow angle maximum at $\theta_{k}$ near zero with decay at large wave numbers as $\sim k^{-4}$, this results in the energy spectrum $E(k) \sim k^{-3}$, which, up to the logarithmic factor, corresponds to the Kraichnan spectrum. For all other angles $\boldsymbol{\epsilon}(k)$ decays proportionally to $k^{-6}$ at large $k$.

We would like to stress once more that the expressions (14) and (15) are resulting from a noncomplete averaging, i.e., the average with respect to coordinates $x_{1 \alpha}, x_{2 \alpha}, y_{0 \alpha}$. In order to get the final result for the energy spectrum it is necessary to average with respect to angles.

Let us assume first that the angle distribution is isotropic. Then, integrating over angles it is easily seen that from the first region (14) we have the following contribution:

$$
E_{1}(k)=2 k \int_{-\theta_{0}}^{\theta_{0}} \epsilon_{1}(k) d \theta_{k} \approx \frac{n}{2 \pi^{2} k^{4} L}\left\langle(\bar{\Gamma} l)^{2}\right\rangle .
$$

Here the factor 2 appears because of two equal contributions from the two regions near $\theta_{k}=0$ and $\theta_{k}=\pi$. Averaging Eq. (15) over angles gives divergence at $\theta \rightarrow 0$ and $\theta \rightarrow \pi / 2$. The main contribution to the energy spectrum comes from the cut-off at small angles $\sim(k L)^{-1}$,

$$
E_{2}(k) \approx \frac{n L^{3}}{3 \pi^{2} k^{4}}\left\langle\left(\Gamma^{\prime}\right)^{2}\right\rangle .
$$

Thus, both regions of angles give contributions of the same order of magnitude. The complete answer for the energy spectrum for isotropic turbulence (i.e., isotropic distribution of discontinuities) is given by the sum of Eqs. (16) and (17),

$$
E(k) \approx \frac{n}{2 \pi^{2} k^{4} L}\left[\left\langle(\bar{\Gamma} l)^{2}\right\rangle+\frac{2 L^{4}}{3}\left\langle\left(\Gamma^{\prime}\right)^{2}\right\rangle\right],
$$

which coincides with the spectrum obtained by Saffman. ${ }^{3}$

In order to find the spectrum in the anisotropic situation one needs to average expressions (14) and (15) with the corresponding distribution function. In numerical experiments anisotropy can be conditioned by box boundaries as well as by anisotropy of the pumping of turbulence. In the case when such ordering is strong enough the spectrum may get some peculiarities originating from nonaveraged spectra given by Eqs. (14) and (15). If the width of the angle distribution function $\Delta \theta$ will be narrower than $\theta_{0}=(k L)^{-1}$, then in the angle cone $\theta_{k}<\Delta \theta$ the energy spectrum $E(k, \theta)$ will have the fall-off $\sim k^{-3}$, i.e., the same power dependence as for the Kraichnan spectrum, with a decrease like $\sim k_{\perp}^{-4}$ perpendicular to the cone direction in accordance with Eq. (15). Note, however, that this asymptotic is only intermediate because $\theta_{0}$ $=(k L)^{-1}$ decreases with increasing $k$. Therefore starting from $k=k^{*}=\left(L \theta_{0}\right)^{-1}$, the average over the angles becomes sensitive relative to the singularities of Eq. (15) that results in the spectrum decreasing proportional to the Saffman falloff. If the influence of anisotropy is not so essential then we should expect the spectrum to be close to the Saffman one, of course, in the case when the main contribution to the spectrum at large $k$ is connected with strong vorticity gradients. The most interesting observation following from the analytical results of this section is that in the very anisotropic case with strong ordering of jumps the sharp angular maximum of the spectrum has the power falloff coinciding with that for the Kraichnan spectrum. While in the isotropic case our result coincides with the Saffman spectrum. In the next section we present results of numerical simulations of decaying 2D turbulence at high-Reynolds numbers. In particular, the appearance of the power law tails in the energy spectrum at large $k$ can be explained by discontinuities, rather than by a cascading process with constant enstrophy. 


\section{NUMERICAL INVESTIGATIONS}

To support the arguments of the previous sections and reveal the direct connection between the formation of the sharp vorticity gradients and the tail of the energy spectrum, we have performed a numerical study of the evolution of decaying 2D turbulence. The 2D Euler equations in the vorticity-stream-function formulation,

$$
\frac{\partial \omega}{\partial t}+\{\omega, \psi\}=\mu_{2 n} \nabla^{2 n} \omega,
$$

are integrated numerically on a double periodic domain by employing a high resolution fully dealiased spectral scheme. $\psi$ is the stream function, $\omega=-\nabla^{2} \psi$ is the vorticity, $\mathbf{v}$ $=\left(v_{x}, v_{y}\right)=\nabla \psi \times \hat{z}$ is the velocity, and the bracket

$$
\{\omega, \psi\} \equiv \mathbf{v} \cdot \nabla \omega=\frac{\partial \omega}{\partial x} \frac{\partial \psi}{\partial y}-\frac{\partial \psi}{\partial x} \frac{\partial \omega}{\partial y} .
$$

In Eq. (19) we have included a hyperviscosity term to keep the integration scheme stable and avoid the so-called bottleneck instability (see, e.g., Ref. 38). Typically we have used $n=3$ and $\mu_{6}=10^{-20}$. This term was observed to decrease the energy by less than $0.002 \%$ and the enstrophy by less than $20 \%$. We verified that the global features of our results were not dependent on the type of viscosity and also kinematic viscosity was applied. In this connection we refer to Ref. 39 for detailed investigations of decaying 2D turbulence with Newtonian viscosity and increasing Reynolds numbers. In the present context we apply the hyperviscosity to allow as wide a dynamical range as possible within the given resolution. The domain size was taken to be unity and the resolution was $2048 \times 2048$. For the time integration we employ a third order stiffly stable scheme. We have chosen the time scale to correspond to $\omega_{0}^{-1}$, where $\omega_{0}$ corresponds to the maximum vorticity, and the spatial scale is the domain size.

As initial conditions we have placed a number of positive and negative vortices randomly on the domain, ensuring that the total circulation is zero. We have employed circular vortices with various profiles from vortex patches (Rankine vortices) to Gaussian vortices. In the simulation run described here we have used 10 vortices of each sign with Gaussian profiles,

$$
\omega(r, \theta)=\omega_{0} \exp \left(-r^{2} / R_{0}^{2}\right),
$$

where $\omega_{0}=1$ for all vortices, while their radii $R_{0}$ are varying in the range $0.02<R_{0}<0.075$. In other runs we have applied different initial conditions with different vortex profiles and different spatial distribution yielding essentially the same results.

In Fig. 1(a) we show the initial vorticity field and Fig. 1 (b) shows the vorticity field at $t=100$, which corresponds to around 8 vortex internal turnover times $\left(T_{v} \equiv 4 \pi / \omega_{0}\right)$. The vorticity field has the typical structure for $2 \mathrm{D}$ turbulence; it consist of large scale structures (vortices) with concentrated vorticity and strongly filamented structures between the vortices. At this time there is still strong dynamics in the flow evolution, with shearing and straining due to mutual interactions of nearby structures. Corresponding to the vorticity field we show the instantaneous one-dimensional energy

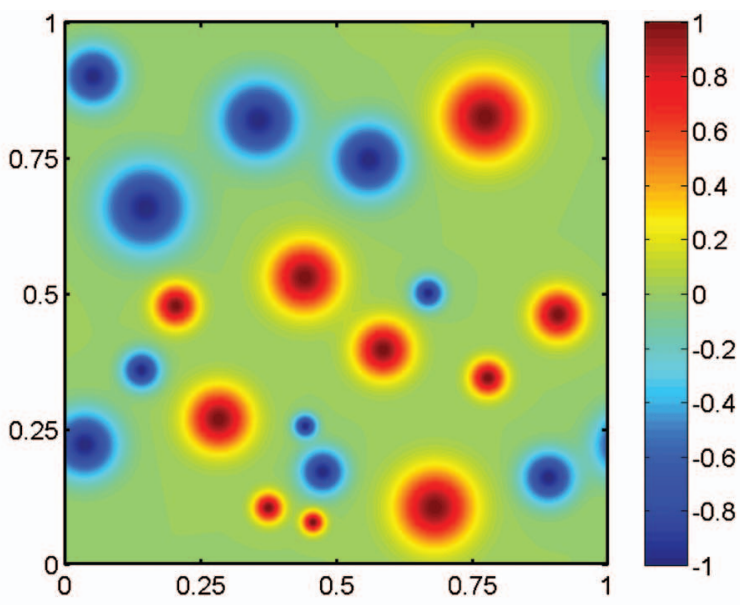

a)

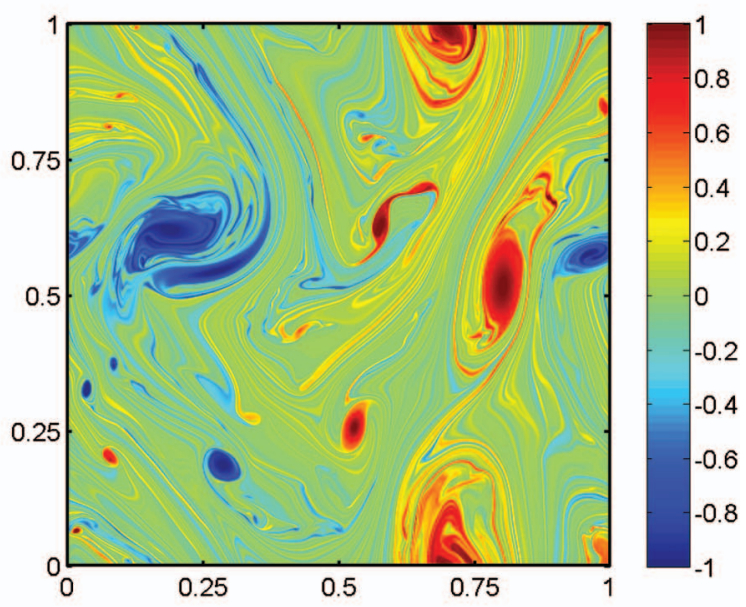

b)

FIG. 1. (Color) (a) Initial vorticity field. (b) Vorticity field at time 100 corresponding to $\approx 8$ vortex turnover times; $\omega_{0}=1$.

spectrum $E(k)$ [total energy: $\left.E=\int_{0}^{\infty} E(k) d k\right]$ in Fig. 2. The spectrum $E(k)$ for $t=0$ in Fig. 2 shows the spectrum of superimposed Gaussian vortices. The spectrum is expanding to the high $k$-values, and at $t=95$ a $k^{-\alpha}$ spectrum has developed at high wave numbers. For the present case $\alpha \sim 3$ as clearly demonstrated in Fig. 2, which show the compensated spectrum $k^{3} E(k)$ is practically constant over almost 2 decades in $k$.

We investigate the details of the dynamics and how the $k^{-\alpha}$ spectrum is generated by examining the divorticity field $\mathbf{B}$ defined in Sec. II. Figure 3 show the length $|\mathbf{B}|$, which is equal to size of the vorticity gradient, $|\nabla \omega|$. It is clear from the figure that very sharp vorticity gradients appears. These are localized in stripes that are mostly along straight lines. The stripes are mainly formed outside the dominating vortex structures, and their formation can be explained by the analysis discussed in Sec. II. Furthermore it is evident that the concentration of the stripes is relatively low. In Fig. 4 we show the time evolution of the maximum value of $|\mathbf{B}|^{2}, B_{\max }^{2}$, i.e., the square of the maximum value of the vorticity gradient. The function is not smooth since we have plotted the 


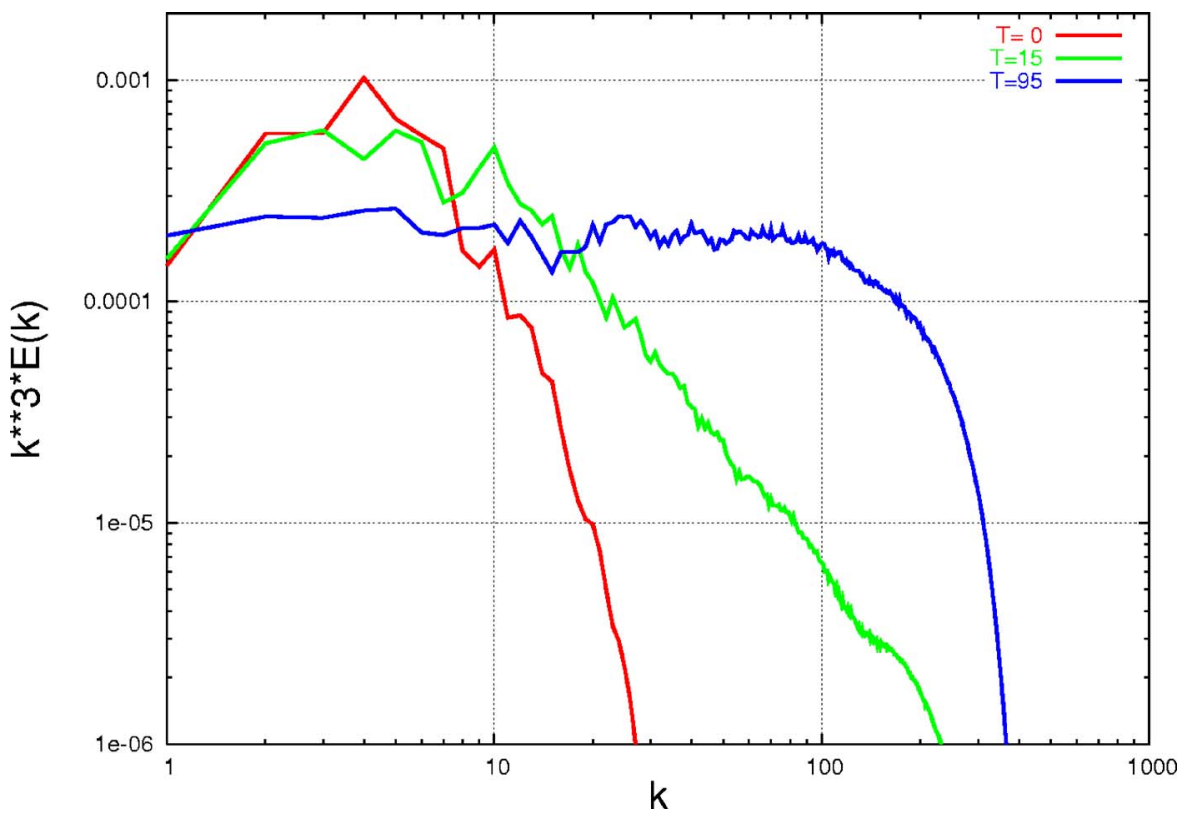

FIG. 2. (Color online) Compensated energy spectrum $k^{3} E(k)$ at different times corresponding to the vorticity field in Fig. 1. absolute maximum within the domain at each time, and jumps in the first derivative appear when the maximum is appearing at a new position. Each smooth part of the curve relates to the development of the maximum value at one point. We observe a rapid growth of $B_{\max }$, which then saturates and decays. The highest value attained by $B_{\max }$ during this simulation approaches 1000 , which with a maximum value of the vorticity $\omega_{0}=1$ corresponds to the width of the filaments $\delta \simeq 0.001$. It is evident that the growth of $|\mathbf{B}|$ is arrested by the hyperviscosity, and indeed the highest value of $B_{\max }$ scales with $\mu_{6}^{-1 / 6}$. The growth of $B_{\max }$ in one point is monitored Fig. 4(b) for a couple of cases. It is observed that the growth initially is exponential, but for later times the growth saturates and becomes weaker than exponential. This evolution certainly respects the bound on the vorticity gradient in Eq. (10).

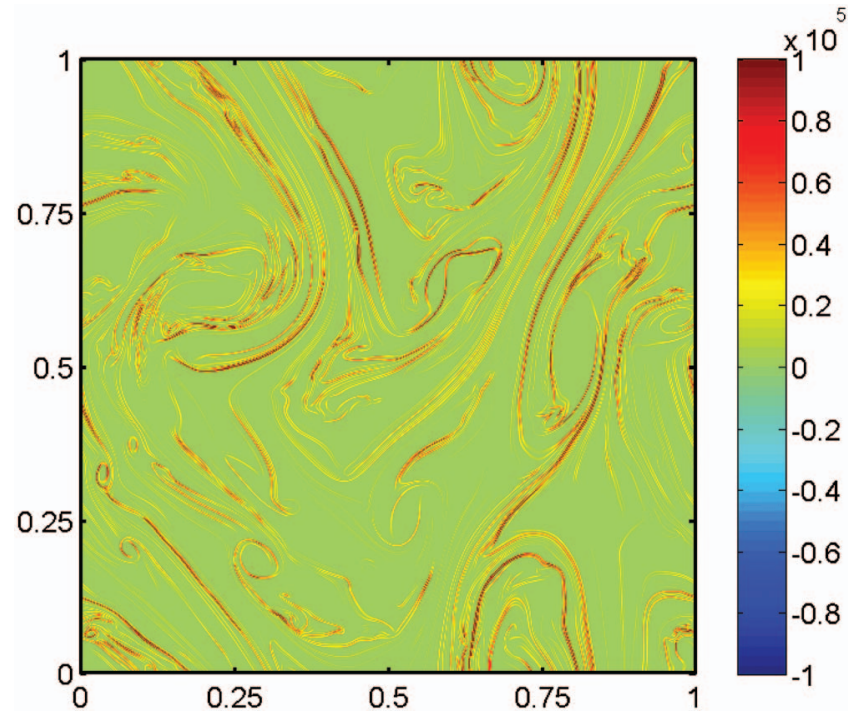

FIG. 3. (Color) The squared length of the divorticity vector $|\mathbf{B}|^{2}$ at time 100 The maximum value is $2.5 \cdot 10^{5}$.
We compare the structure of the divorticity field with the high pass filtered vorticity field shown in Fig. 5. The very similar structure of the high pass filtered vorticity field and the divorticity field strongly suggests that the vorticity gradients are responsible for the large- $k$ part of the spectrum, i.e., the $k^{-\alpha}$ part. This is further supported by the structure of the Weiss field, ${ }^{22}$ which is defined by $W=1 / 4\left(\sigma^{2}-\omega^{2}\right)$, where $\sigma^{2}=-\operatorname{det}[\hat{S}]=\left(\partial_{x} v_{x}-\partial_{y} v_{y}\right)^{2}+\left(\partial_{x} v_{y}+\partial_{y} v_{x}\right)^{2}$ is the square strain rate, i.e., minus the determinant of the stress tensor defined in Eq. (5). The Weiss field, which is depicted in Fig. 6 , separates the flow into regions with $W>0$ that are strain dominated ( $h$ regions) and regions with $W<0$ that are vorticity dominated ( $e$ regions). Comparing Figs. 3 and 6, we observe that the vorticity gradient stripes are aligned with the contours of $W$ in the strain dominated regions, $W>0$, mainly at the edge of the vortex structures and in between the structures. A careful inspection, however, reveals that in the stripes $W<0$, i.e., vorticity dominates. This is in line with the original arguments of Weiss (see also Refs. 21 and 23) that vorticity gradients will tend to concentrate in the strain dominated regions. In particular in the work of Chen et al. ${ }^{23}$ it was demonstrated that the dynamics leading to the enstrophy cascade in driven 2D turbulence is most significant in strain dominated regions. We note that the Weiss field is given by the determinant of the velocity gradient tensor, and it is possible to express the "linearized" evolution (in a frozen velocity field) of the divorticity as (e.g., Ref. 21)

$$
|\mathbf{B}| \propto \exp (\sqrt{W} t)
$$

which shows that in the hyperbolic regions the divorticity will grow exponentially, while it will have an oscillatory behavior in the elliptic regions.

The spectra we have observed are characterized by having the exponent close to $\alpha=3$. Thus, with reference to Sec. III this corresponds to the spectrum in the anisotropic regime where the stripes of vorticity gradients are near straight lines. Indeed in Fig. 3 we see that the stripes are close to straight 
a)

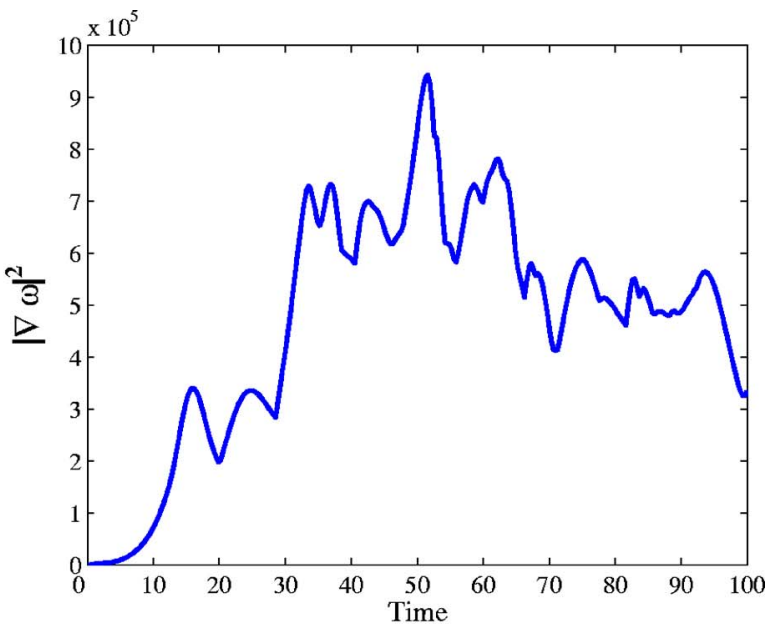

b)

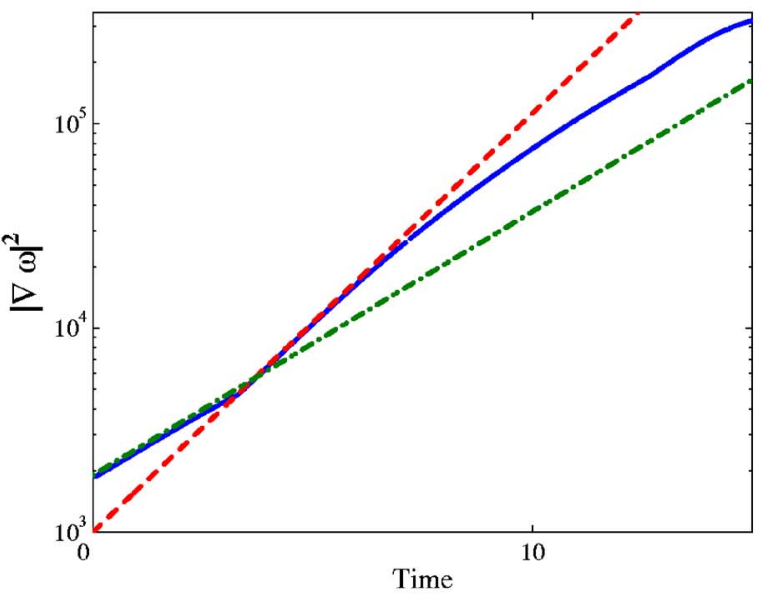

FIG. 4. (Color online) (a) The temporal evolution of the squared maximum of the divorticity equal to the squared maximum vorticity gradient $|\nabla \omega|^{2}$. (b) Close-up of the growth of the maximum in two cases.

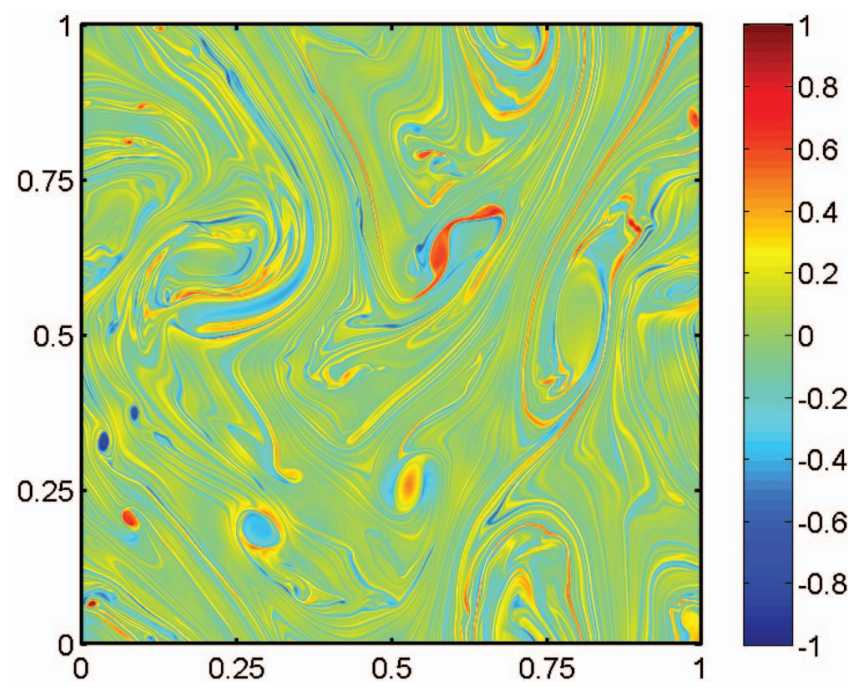

FIG. 5. (Color) High pass filtered vorticity field from Fig. 1(b), i.e., the vorticity at mode numbers $k>10$ is plotted. The amount of energy in the filtered field is about $1 \%$ of the total energy.

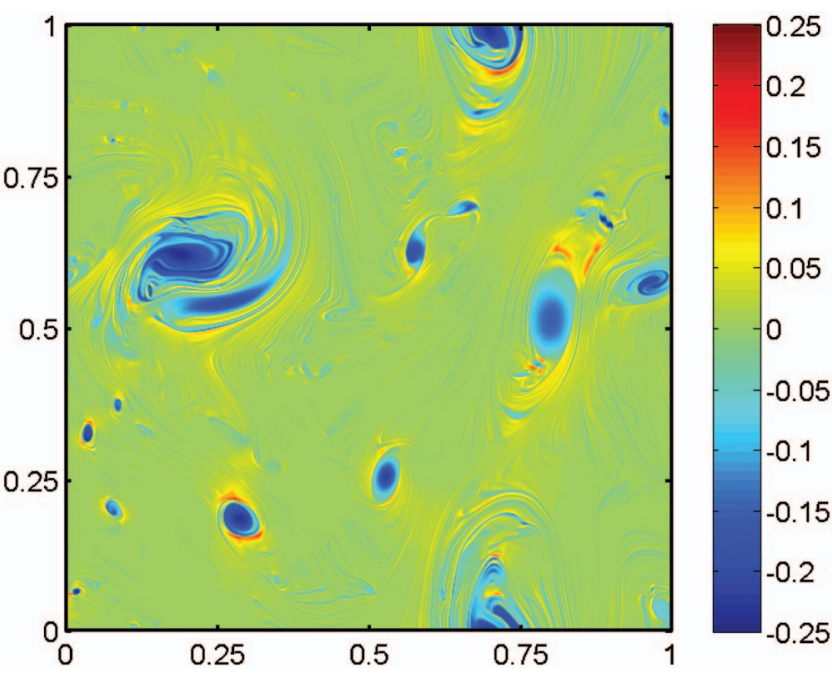

FIG. 6. (Color) Weiss field for the vorticity field in Fig. 1(b). Red designates strain dominated $(h-)$ regimes. Blue designates vorticity dominated $(e-)$ regimes.

lines and the spectrum is in keeping with the expectations. To illustrate the anisotropic nature of the spectrum directly we plot in Fig. 7 the two-dimensional spectrum, $\epsilon\left(k_{x}, k_{y}\right)$. We observe a clear anisotropy that becomes particular apparent in the compensated spectrum in Fig. 7(b), which is obtained by subtracting the angle average of $\epsilon\left(k_{x}, k_{y}\right)$ [i.e., $\left.(2 \pi)^{-1} \int_{0}^{2 \pi} \epsilon\left(k_{x}, k_{y}\right) d \theta\right]$. Here we observe a clear angular structure with significant "jets" pointing in specific directions. Similar jet-like structures in $k$-space have been anticipated by Dubrulle et $a l^{40}$ based on a stochastic distortion approach for strongly nonlocal interactions (see also Ref. 41). We should emphasize that the spectra obtained here are instantaneous spectra obtained at one time and for one realization. Ensemble averaged spectra may be expected to show a much higher degree of angle isotropy.

\section{CONCLUDING REMARKS}

We have performed a detailed investigation of the relation between turbulent spectra and sharp vorticity gradients in 2D turbulent flows. First, we have demonstrated that the $k$-behavior of the spectra generated by sharp vorticity gradients, based on the compressible advection of divorticity, depends significantly on the anisotropy of the spectra. If the angular distribution in the spectrum has one or more very sharp peaks, then the one-dimensional spectrum has a tail falling-off like $k^{-3}$ at large $k$, which resembles the Kraichnan spectrum, derived from spectral cascade arguments. In the opposite case of an isotropic smooth angular dependence the spectrum has an asymptotic behavior $k^{-4}$ as for the Saffman spectra. These arguments allow us to suggest an interpretation of the many numerical experiments where the spectral exponent varies in the whole interval between -3 and -4 . For instance, in the simulations by Ohkitani ${ }^{21}$ the $e$ regions, because of their geometry, would mainly contribute to the isotropic component of the spectrum, which explain the Saffman exponents for the observed spectrum in these regions. For $h$ regions the situation is different; these regions contain stretched vorticity level lines and their contribution to the 

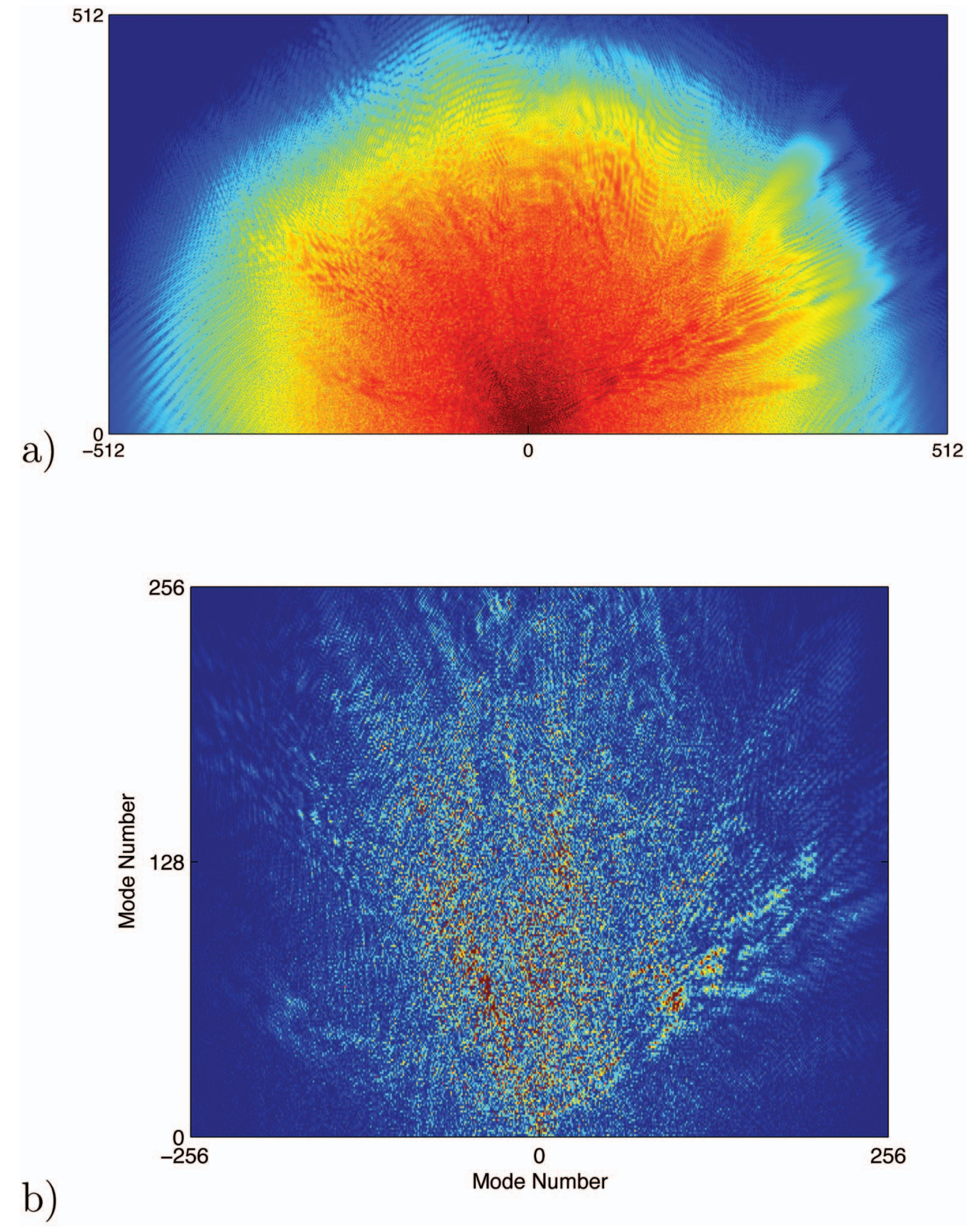

FIG. 7. (Color) (a) 2D energy spectrum $\epsilon\left(k_{x}, k_{y}\right)$, logarithmic scale. (Red designates the highest positive values, blue the negative values.) (b) The $2 \mathrm{D}$ energy spectrum $\epsilon\left(k_{x}, k_{y}\right)$ compensated by subtracting the angle averaged spectrum, linear scale. (Red designates the highest values, deep blue is "zero.")

spectrum would be expected to be very anisotropic. This is why these regions contribute to the $k$ behavior for the Kraichnan spectrum. A similar situation takes place in our present investigations in comparison to previous investigations, ${ }^{25,26}$ where the spectra were isotropic resulting in spectral exponents like for the Saffman spectrum. In the simulations presented in the present paper we have very strong vorticity gradients concentrated on narrow stripes and therefore the exponent is close to that for the Kraichnan spectrum. Employing a filtering of the vorticity field suggests that the tail of the spectrum originates from the sharp vorticity gradients. We should emphasize that in the derivation of the spectra from the spatial structure of the vorticity we have inherently assumed that the distribution of sharp vorticity gradient stripes are dilute in the sense that they may be considered independent and the spectrum can thus be constructed by summing up their individual contributions. Breaking of this assumption may lead to a different spectral form tending to become more shallow, e.g., as demonstrated by Vassilicos and Hunt. ${ }^{20}$

The energy spectrum is one of the statistical characteristics of the turbulent state. In order to get more information about such states one needs to investigate higher moments in $k$-space, especially if it concerns $k$-jets. As it was shown in Ref. 40 (see also Ref. 41) the higher moments are very sensitive to such distributions. However, this problem is beyond the scope of the present paper, and will be the subject of future investigations. 
The strong amplification of the divorticity of up to one thousand times is one of the main results of our simulations. Fitting of the temporal behavior for the maxima demonstrates an initial exponential increase with saturation at later times. This dependence is far from the double exponential growth given by the estimate in Eq. (10). In conclusion, we stress that this amplification has a natural explanation in the compressibility of the mapping (8) providing the transfer from the Eulerian description to the system of movable curvilinear divorticity lines as described in Sec. II.

Finally, we note that similar arguments as presented in this paper on the connection between the Kraichnan and Saffman spectra, can also be applied for turbulence of water (gravity) waves, where wedges represent surface singularities as well as for acoustic turbulence where shocks play the role of singularities. ${ }^{42}$

\section{ACKNOWLEDGMENTS}

This work was supported by the Danish Center for Scientific Computing and partially by INTAS.

The work of E.A.K. was also supported by the RFBR (Grant No. 06-01-00665). E.A.K. wishes to thank the Riso National Laboratory, where this work was initiated, for its kind hospitality during the visit. J.J.R. thanks the Landau Institute for kind hospitality during a later visit, where the work was almost finalized.

${ }^{1}$ R. H. Kraichnan, "Inertial ranges in 2D turbulence," Phys. Fluids 11, 1417 (1967).

${ }^{2}$ R. H. Kraichnan, "Inertial range transfer in two- and three-dimensional turbulence," J. Fluid Mech. 47, 525 (1971); “Kolmogorov's inertial-range theories," ibid. 62, 305 (1974).

${ }^{3}$ P. G. Saffman, "On the spectrum and decay of random 2D vorticity distributions at large Reynolds number," Stud. Appl. Math. 50, 377 (1971).

${ }^{4} \mathrm{~W}$. Wolibner, "Un théorème sur l'existence du movement plan d'un fluide parfait, homogène, incompressible, pedant un temp infiniment long," Math. Z. 37, 698 (1933).

${ }^{5}$ T. Kato, "On classical solutions of two-dimensional non-stationary Euler equation," Arch. Ration. Mech. Anal. 25, 189 (1967).

${ }^{6}$ V. I. Yudovich, "Nonstationary flow of an ideal incompressible liquid," Zh. Vychisl. Mat. Mat. Fiz. 3, 1032 (1963) [J. Math. Numer. Phys. Math. 6, 1032 (1965)]; "On the loss of smoothness of the solutions of the Euler equations and the inherent instability of flows of an ideal fluid," Chaos $\mathbf{1 0}$, 705 (2000).

${ }^{7}$ H. A. Rose and P. L. Sulem, "Fully developed turbulence and statistical mechanics," J. Phys. (France) 39, 441 (1978).

${ }^{8}$ C. Sulem and P. L. Sulem, "The well-posedness of two-dimensional ideal flow," J. Mec. Theor. Appl. Special Issue S1, 217 (1983).

${ }^{9}$ O. A. Ladyzhenskaya, The Mathematical Theory of Viscous Incompressible Flow (Gordon and Breach, New York, 1969).

${ }^{10} \mathrm{O}$. A. Ladyzhenskaya, "Mathematical analysis of Navier-Stokes equations for incompressible liquids," Annu. Rev. Fluid Mech. 7, 249 (1975).

${ }^{11}$ U. Frisch, T. Matsumoto, and J. Bec, "Singularities of Euler flows? Not out of the blue!" J. Stat. Phys. 113, 761 (2003).

${ }^{12}$ T. Matsumoto, J. Bec, and U. Frisch, "The analytic structure of 2D Euler flows at short times," Fluid Dyn. Res. 36, 221 (2005).

${ }^{13} \mathrm{~J}$. C. McWilliams, "The emergence of isolated coherent vortices in turbulent flow," J. Fluid Mech. 146, 21 (1984).

${ }^{14} \mathrm{~S}$. Kida, "Numerical simulations of two-dimensional turbulence with highsymmetry," J. Phys. Soc. Jpn. 54, 2840 (1985).

${ }^{15}$ M. E. Brachet, M. Meneguzzi, and P. L. Sulem, "Small scale dynamics of high Reynolds number two-dimensional turbulence," Phys. Rev. Lett. 57, 683 (1986).

${ }^{16}$ R. Benzi, S. Patarnello, and P. Santangelo, "On the statistical properties of two-dimensional decaying turbulence," Europhys. Lett. 3, 811 (1987).

${ }^{17}$ B. Legras, B. Santangelo, and R. Benzi, "High resolution numerical ex- periments for forced of two-dimensional turbulence," Europhys. Lett. 5, 37 (1988); B. Santangelo, R. Benzi, and B. Legras, "The generation of vorticers in high-resolution two-dimensional decaying turbulence and the influence of initial conditions on the breaking of self-similarity," Phys. Fluids A 1, 1027 (1989).

${ }^{18}$ A. D. Gilbert, "Spiral structures and spectra in two-dimensional turbulence," J. Fluid Mech. 193, 475 (1988).

${ }^{19}$ H. K. Moffatt, "Spiral structures in turbulent flow," in New Approaches and Concepts in Turbulence, edited by Th. Dracos and A. Tsinober (Birkhäuser, Basel, 1993), p. 121.

${ }^{20}$ J. C. Vassilicos and J. C. R. Hunt, "Fractal dimensions and spectra of interfaces with applications to turbulence," Proc. R. Soc. London, Ser. A 435, 505 (1991).

${ }^{21} \mathrm{~K}$. Ohkitani, "Wave number space dynamics of enstrophy cascades in a forced two-dimensional turbulence," Phys. Fluids A 3, 1598 (1991).

${ }^{22}$ J. Weiss, "The dynamics of enstrophy transfer in two-dimensional hydrodynamics," Physica D 48, 273 (1991).

${ }^{23}$ S. Chen, R. E. Ecke, G. L. Eyink, X. Wang, and Z. Xiao, "Physical mechanism of the two-dimensional enstrophy cascade," Phys. Rev. Lett. 91, 214501 (2003).

${ }^{24}$ M. Do-Khac, C. Basdevant, V. Perrier, and K. Dang-Tranc, "Wavelet analysis of 2D turbulent fields," Physica D 76, 252 (1994).

${ }^{25}$ A. H. Nielsen, X. He, J. J. Rasmussen, and T. Bohr, "Vortex merging and spectral cascade in two-dimensional flows," Phys. Fluids 8, 2263 (1996).

${ }^{26}$ J. J. Rasmussen, A. H. Nielsen, and V. Naulin, "Dynamics of vortex interaction in two-dimensional flows," Phys. Scr. 98, 29 (2002).

${ }^{27}$ N. K.-R. Kevlahan and M. Farge, "Vorticity filaments in two-dimensional turbulence: Creation, stability, and effect," J. Fluid Mech. 346, 49 (1997).

${ }^{28}$ D. G. Dritschel, "Vortex properties of 2D turbulence," Phys. Fluids A 5, 984 (1993); "A general theory for two-dimensional vortex interactions," J. Fluid Mech. 293, 269 (1995).

${ }^{29}$ B. Legras and D. G. Dritschel, "Vortex stripping and the generation of high vorticity gradients in two-dimensional flows," Appl. Sci. Res. 51, 445, (1993).

${ }^{30}$ E. A. Kuznetsov and V. P. Ruban, "Hamiltonian dynamics of vortex lines in hydrodynamic-type systems," Pis'ma Zh. Eksp. Teor. Fiz. 67, 1015 (1998) [JETP Lett. 67, 1076 (1998)]; "Hamiltonian dynamics of vortex and magnetic lines in hydrodynamic type systems," Phys. Rev. E 61, 831 (2000).

${ }^{31}$ E. A. Kuznetsov, "Vortex line representation for flows of ideal and viscous fluids,” Pis'ma Zh. Eksp. Teor. Fiz. 76, 406 (2002) [JETP Lett. 76, 346 (2002)].

${ }^{32}$ E. A. Kuznetsov, T. Passot, and P. L. Sulem, "Compressible dynamics of magnetic field lines for incompressible magnetohydrodynamic flows," Phys. Plasmas 11, 1410 (2004).

${ }^{33}$ Y. Kimura and J. R. Herring, "Gradient enhancement and filament ejection for a nonuniform elliptic vortex in two-dimensional turbulence," J. Fluid Mech. 493, 43 (2001).

${ }^{34}$ G. E. Volovik and V. P. Mineev, "Investigation of singularities in super fluid HE-3 and liquid-crystals by homotopic topology methods," Zh. Eksp. Teor. Fiz. 72, 2256 (1977) [Sov. Phys. JETP 45, 1186 (1977)].

${ }^{35}$ E. A. Kuznetsov and V. P. Ruban, "Collapse of vortex lines in hydrodynamics," Zh. Eksp. Teor. Fiz. 118, 853 (2000) [J. Exp. Theor. Phys. 91, $775(2000)]$.

${ }^{36}$ V. A. Zheligovsky, E. A. Kuznetsov, and O. M. Podvigina, "Numerical modeling of collapse in ideal incompressible hydrodynamics," Pis'ma Zh. Eksp. Teor. Fiz. 74, 402 (2001) [JETP Lett. 74, 367 (2001)].

${ }^{37}$ E. A. Kuznetsov, O. M. Podvigina, and V. A. Zheligovsky, "Numerical evidence of breaking of vortex lines in an ideal fluid," in Fluid Mechanics and its Applications, Vol. 71, edited by K. Bajer and H. K. Moffatt (Kluwer, Dordrecht, 2003), pp. 305-316.

${ }^{38}$ G. Falkovich, "Bottleneck phenomenon in developed turbulence," Phys. Fluids 6, 1411 (1994)

${ }^{39}$ J. R. Chasnov, "On the decay of two-dimensional homogeneous turbulence," Phys. Fluids 9, 171 (1997).

${ }^{40}$ B. Dubrulle, J.-P. Laval, S. V. Nazarenko, and O. Zaboronski, "A model for rapid stochastic distortions of small-scale turbulence," J. Fluid Mech. 520, 1 (2004).

${ }^{41}$ S. V. Nazarenko and J.-P. Laval, "Non-local two-dimensional turbulence and Batchelor's regime for passive scalars," J. Fluid Mech. 408, 301 (2000).

${ }^{42}$ E. A. Kuznetsov, "Turbulent spectra generated by singularities," Pis'ma Zh. Eksp. Teor. Fiz. 80, 92 (2004) [JETP Lett. 80, 83 (2004)]. 